\title{
Du discours sociologique à l'imaginaire nationaliste: Barrès et le bon usage des boucs émissaires
}

\author{
Jean-Michel Wittmann
}

\section{(2) OpenEdition} Journals

Édition électronique

URL : http://journals.openedition.org/studifrancesi/9767

DOI : 10.4000/studifrancesi.9767

ISSN : 2421-5856

Éditeur

Rosenberg \& Sellier

\section{Édition imprimée}

Date de publication : 1 août 2017

Pagination : 261-269

ISSN : 0039-2944

\section{Référence électronique}

Jean-Michel Wittmann, « Du discours sociologique à l'imaginaire nationaliste: Barrès et le bon usage des boucs émissaires ", Studi Francesi [En ligne], 182 (LXI | II) | 2017, mis en ligne le 01 août 2018, consulté le 06 janvier 2021. URL : http://journals.openedition.org/studifrancesi/9767 ; DOI : https:// doi.org/10.4000/studifrancesi.9767

\section{(c)}

Studi Francesi è distribuita con Licenza Creative Commons Attribuzione - Non commerciale - Non opere derivate 4.0 Internazionale. 


\title{
Du discours sociologique à l'imaginaire nationaliste: Barrès et le bon usage des boucs émissaires
}

\begin{abstract}
The three parts of the scapegoat cognitive mechanism (crisis, aggressiveness to an individual or a minority group, catharsis which restores even strengthens the cohesion of the social group) are present in Barrès' novels, especially in the trilogy Le Roman de l'énergie nationale. The presence of scapegoats in these novels presents a number of peculiarities, that is why it cannot be interpreted using an external interpretative model, like René Girard's. In regards to the historical context, it takes on a different perspective. The scapegoat representation in these novels helped Barrès develop a nationalist ideology, during the time of the Dreyfus' affair, as well as his observation of a social crisis which he calls uprooting. This representation connects to the rising sociological thoughts, because the scapegoat has been used by Durkheim to explain anti-Semitism. However, Barrès and Durkheim diverge: the sociologist gives a demystifying explanation, whereas the nationalist novelist uses the scapegoat to introduce his readers to the necessity of defending the social body against invaders.
\end{abstract}

La figure et, plus généralement, le «programme» du bouc émissaire, articulé autour de trois éléments (la crise, le déplacement de l'agressivité sur un individu ou un groupe minoritaire, la catharsis susceptible de restaurer voire de renforcer la cohésion du groupe social ${ }^{1}$ ), sont présents dans les œuvres romanesques de Maurice Barrès, notamment dans la trilogie du Roman de l'énergie nationale. Dans Les Déracinés (1897), l'élimination d'un des jeunes Lorrains, Honoré Racadot, qui favorise finalement la socialisation de ses camarades, peut être interprétée à l'aide de ce mécanisme cognitif. Dans Leurs figures (1902), il est fait directement référence à la figure du bouc émissaire, au moment de raconter le suicide du baron de Reinach, érigé en «victime expiatoire» $\left(L f, 1101^{2}\right)$. La présence de figures émissariales dans le texte barrésien présente néanmoins certaines particularités qui engagent à ne pas l'interpréter à partir d'un modèle interprétatif externe et postérieur, comme peut l'être celui de René Girard'. La présence explicite du bouc émissaire dans l'œuvre de Barrès intervient, en effet, dans un contexte historique défini. C'est en 1899, dans La Réparation, que Clemenceau, évaluant «le rôle historique de l'affaire Dreyfus», constate que «sur ce bouc émissaire du judaïsme, tous les crimes anciens se trouvent représentativement accumulés» ${ }^{4}$. La même année, Émile Durkheim explique la réaction de la foule française lors de la condamnation de Dreyfus en 1894, en mettant en avant la notion de «victime expiatoire» ${ }^{5}$, reprise telle quelle dans Leurs figures. La représentation

(1) Voir G. ERner, Expliquer l'antisémitisme, Paris, Presses Universitaires de France, «Quadrige», 2012.

(2) Les références de page des citations des Déracinés $(D)$ et de Leurs figures $(L f)$ seront directement indiquées entre parenthèses à la suite de ces citations, en renvoyant à l'édition suivante: M. BARRÈs, Romans et Voyages, éd. V. Rambaud, vol. 1, Paris, Robert Laffont, «Bouquins», t. 1, 1994.

(3) Voir R. Girard, Le Bouc émissaire, Paris, Grasset, 1982.

(4) G. Clemenceau, La Réparation, Paris, 1899, p. 296.

(5) Voir infra, et note 16. 
du bouc émissaire dans les romans de Barrès participe donc de la construction de la doctrine nationaliste durant l'Affaire, articulée autour du constat d'une crise sociale dont rend compte la notion de déracinement, dans Le Roman de l'énergie nationale, et au-delà de la fiction, dans Scènes et doctrines du nationalisme. Du même coup, elle se trouve aussi croiser, tout en s'y opposant, le discours de la sociologie naissante qui, avec Durkheim, s'empare au même moment de la figure du bouc émissaire pour rendre compte de certains mécanismes sociaux et, en particulier, de l'antisémitisme, en insistant sur la notion de crise ${ }^{6}$.

Alors que dans Leurs figures, la référence explicite à la figure du bouc émissaire peut apparaître comme une réponse ironique et critique à l'interprétation de l'Affaire développée par Clemenceau et par Durkheim, le schéma d'émissarisation, dans Les Déracinés, s'inscrit dans le texte de manière transparente, sans donner lieu à un commentaire explicite de la part d'un auteur qui, tout au long de ses romans à thèse, n'hésite pourtant pas à interrompre le récit pour en commenter le déroulement, voire en tirer des conclusions ${ }^{7}$. Cette différence de statut s'explique par la chronologie. Certes, dans Le Roman de l'énergie nationale, dont la diégèse se déroule entre l'automne 1879 (année où les jeunes gens préparent le bac au lycée de Nancy) et décembre 1893 (période marquée par le scandale de Panama), Barrès se livre à «une reconstruction d'une période de l'histoire de France selon les normes politiques de la période suivante», dominée par les débats liés à l'Affaire ${ }^{8}$. Pour autant, il ne faut pas oublier que le premier volume a été rédigé avant le grand affrontement autour de la révision du procès Dreyfus. L'Appel au soldat et Leurs figures, en revanche, ont été écrits au plus fort de la bataille par un Barrès qui fige alors sa doctrine, sans se priver de relire Les Déracinés à la lumière du jeu d'oppositions qui structure désormais le discours nationaliste'. S'il n'y a pas de solution de continuité, au plan idéologique, entre le premier volet de la trilogie et les deux suivants, l'inscription du schéma d'émissarisation dans Les Déracinés, en revanche, est indépendante des commentaires de Clemenceau ou de Durkheim sur le rôle de l'antisémitisme dans l'Affaire. Elle n'en est pas moins représentative de l'ambition proprement sociologique du romancier, saluée par la critique de son époque. À la publication des Déracinés, Barrès est présenté comme «un homme d'imagination, [...] un historien et un sociologue» ${ }^{10}$, et son livre, comme «un roman expérimental» doublé d'une «démonstration de sciences sociales» ${ }^{11}$, enfin comme «un roman sociologique» ${ }^{12}$.

(6) Voir Expliquer l'antisémitisme cit., en particulier «Chapitre 2. Naissance du modèle du bouc émissaire», pp. 43-82. Si le constat d'une crise sociale et politique, avec la notion d'anomie, est central chez Durkheim, celui-ci ne met cependant pas en cause le rationalisme des Lumières, la démocratie ou le développement de l'individualisme, au contraire des nationalistes comme Barrès: voir C.A. GOLDBERG, Introduction to Emile Durkheim's "Antisemitism and Social Crisis", «Sociological Theory», vol. XXVI, 4, pp. 299-323 (en particulier, p. 302).

(7) Compte tenu du fait que non seulement Barrès interrompt le récit pour le commenter, mais fait allusion à sa propre situation d'homme public en évoquant son mandat à la chambre, dans Leurs figures, la distinction introduite par la narratologie entre «auteur» et «narrateur» n'a pas lieu d'être appliquée au Roman de l'énergie nationale.

(8) Voir Z. Sternhell, Maurice Barrès et le nationalisme français, Paris, Éditions Complexe, 1985, p. 110.

(9) Sur la manière dont le déroulement de l'affaire Dreyfus a pu orienter et modifier progressivement la réception des Déracinés, et sur le rôle que Barrès lui-même a pu jouer dans ce processus, voir E. GoDO et J.-M. WitTmann, «Introduction», dans M. Barrès, Les Déracinés, Paris, Honoré Champion, 2004, pp. 66-67.

(10) R. Doumic, "Les Déracinés” de M. Maurice Barrès, «La Revue des deux mondes», 15 novembre 1897.

(11) M. Collière, Maurice Barrès: “Les Déracinés”, «Mercure de France», janvier 1898.

(12) L. Bellugou, "Les Déracinés" de M. Barrès et le professeur Bouteiller, «Mercure de France», février 1898. 
Il apparaît en effet que les éléments constitutifs du schéma émissarial: la crise, l'élimination de l'élément censément perturbateur, la catharsis, entrent pour ainsi dire naturellement en résonance avec l'imaginaire barrésien, avant même de rencontrer les points saillants de sa doctrine. Dès ses premiers livres, l'univers romanesque barrésien a en effet cristallisé autour d'un certain nombre de motifs, au premier rang desquels figurent l'empoisonnement, l'invasion, la dégradation, qui structurent sa vision du moi autour d'une opposition entre santé et maladie, mais aussi entre intégrité et décomposition, ou dissolution. Tout naturellement, chez un écrivain dont la conception de la société et de la nation est essentiellement organiciste, ces mêmes éléments ont favorisé la construction de ce que l'on peut appeler un imaginaire nationaliste, notamment dans Le Roman de l'énergie nationale. Barrès lui-même établit une équivalence entre le moi et la nation. Il souligne dans Les Déracinés que la France n'a «pas subi seulement un Sedan militaire, politique, financier, industriel», mais «encore un Sedan intellectuel»: «Avec l'intégrité du territoire à reconstituer, il y a aussi l'intégrité psychologique à sauvegarder» $(D, 661)$. La France selon Barrès est minée par une maladie: la décadence, ou si l'on préfère - le terme de décadence étant finalement peu présent dans le vocabulaire barrésien ${ }^{13}$ - l'individualisme, qui en est tout à la fois la cause et le symptôme, comme l'avait magistralement établi son ami Paul Bourget, dès 1881, dans sa célèbre étude sur Baudelaire ${ }^{14}$. En crise, la France représentée par Barrès est, comme le souligne le titre du chapitre IX des Déracinés, «dissociée et décérébrée». Recouvrer son intégrité suppose pour elle de combattre à la fois les ennemis de l'intérieur (les organismes qui cessent de se mettre au service de l'organisme national, autrement dit: les individus déracinés) et les ennemis de l'extérieur (les corps étrangers susceptibles de corrompre l'organisme): à ce prix seulement, la catharsis peut opérer et la guérison, devenir envisageable. Tout semble donc bien préparer Barrès à fantasmer sur le schéma émissarial, de la structure propre de son univers imaginaire à son ambition de dépeindre et d'analyser des faits sociaux afin de rendre compte, en romancier sociologue, des dysfonctionnements qui affaiblissent selon lui la France de son temps.

Principale péripétie dans la diégèse des Déracinés, le meurtre d'Astiné Aravian par Racadot et son complice Mouchefrin, qui conduit à l'exécution du premier, installe au cœur du roman le programme du bouc émissaire. Les trois moments du programme (la crise, le déplacement de l'agressivité sur un individu ou un groupe minoritaire, la catharsis qui renforce finalement la cohésion du groupe social) sont bien présents, même si leur articulation est en partie masquée. En apparence, il s'agit simplement d'un crime crapuleux. Pressé par ses créanciers, Racadot, qui a dilapidé son maigre héritage en fondant un journal, tue cette riche étrangère afin de lui dérober ses bijoux. De ce point de vue, Racadot ne peut pas être considéré comme un bouc émissaire. Le roman invite cependant à considérer le crime comme un fait social. Coupable, mais pas encore démasqué, Racadot est en effet l'auteur d'une conférence où, dans la lignée du darwinisme social, il présente la lutte pour la vie comme une loi fondamentale de l'existence. Inscrit dans cette logique de lutte pour la vie, le crime de Racadot manifeste, sous sa forme la plus extrême, le triomphe de l'individualisme

(13) Le mot «décadence» n'est pas utilisé dans Les Déracinés; il apparaîtra, en revanche, dès le premier chapitre du volume suivant, L'Appel au soldat, sous la plume de Roemerspacher, amené à noter, lors d'un séjour outre Rhin, que les Allemands «tiennent notre décadence pour un fait» $(\mathrm{Lf}, \mathrm{773})$. Pour autant, ce que M. WinOcK décrit comme l'antienne de «l'éternelle décadence» dans Nationalisme, antisémitisme et fascisme en France, Paris, Seuil, 1999, «Points», p. 99, n’en est pas moins présent dans Les Déracinés.

(14) Voir P. Bourget, «Baudelaire, III: Théorie de la décadence» (1881), dans Essais de psychologie contemporaine, Paris, Gallimard, 1993, «Tel», pp. 13-18. 
dans la société contemporaine. Quant au titre du dernier chapitre, «Déraciné, décapité», il énonce explicitement le rapport qui unit la crise sociale (le déracinement) et l'exécution de Racadot.

S'il n'est pas absent du roman, le point de vue moral s'efface vite derrière un point de vue social. L'un des jeunes Lorrains, François Sturel, se trouve avoir aperçu Racadot, Mouchefrin et Astiné le soir de l'assassinat, à Billancourt, sur les lieux mêmes du crime. Son témoignage pouvant établir la culpabilité de Racadot, Sturel se lance alors dans une controverse avec ses camarades, qui conduit à considérer le criminel et son complice comme les victimes d'un certain ordre social. Avant même le chapitre consacré au crime d'Astiné, l'auteur souligne déjà que la situation de Racadot a «une valeur historique», tout en énonçant une loi sociale: «Le mécanisme instinctif de cette collectivité tend à expulser les Racadot, les Mouchefrin, à les rejeter dans le prolétariat, à les dégrader» $(D, 686)$. C'est ensuite Roemerspacher qui, sans exonérer les criminels de leur responsabilité, admet «que la société, dans ses rapports avec Racadot, avec Mouchefrin, ne s' [est] pas conduite selon le principe kantien... Si l'individu doit servir la société, celle-ci doit servir l'individu» $(D, 744)$. Ainsi esquissé, le schéma émissarial se trouve inscrit avec netteté dans le roman quand Sturel en vient à introduire la notion de sacrifice, dans le contexte des funérailles nationales de Victor Hugo, qui lui fournissent l'occasion de parfaire sa socialisation. Dans ce moment particulier où le peuple français est dans la rue, où «la sève nationale est en émoi», Sturel a l'intuition que «des millions d'êtres sont sacrifiés, voire damnés, uniquement parce que la nature en fera, dans ses abîmes, comme dit Hugo, quelque chose de grand» $(D, 730)$. Il établit alors un parallèle entre la situation de Racadot et Mouchefrin, et celle des badauds qui, dans la cohue, sont «jetés à terre, foulés, sacrifiés» $(D, 737)$, au moment exact où «la magnifique cérémonie civique» lui révèle la profondeur du «mot de Roemerspacher [...]: “Je suis un homme social”» $(D, 737)$. Après l'exécution de Racadot, il tire cette leçon de l'événement: «Dans l'essai de notre petite bande pour se hausser, il était certain qu'il y aurait du déchet. Racadot, Mouchefrin, sont notre rançon, le prix de notre perfectionnement. Je hais leur crime, mais je persiste à tenir, par rapport à moi, comme des sacrifiés» $(D, 744)$. Ainsi, le crime crapuleux apparaît finalement comme un sacrifice nécessaire, qui produit un bénéfice social. L'auteur se charge lui-même de reprendre cette conclusion, en liant à nouveau l'acte criminel et la socialisation réussie de certains des jeunes gens: «Par un brutal accident, ils avaient pris avec la société le contact direct qu'ils avaient tant cherché» $(D, 745)$.

Le schéma émissarial n'est donc pas seulement inscrit dans la diégèse du roman. Un discours de type sociologique qui vise à expliquer le «mécanisme instinctif», non pas seulement du groupe des jeunes Lorrains, mais bien de la société entière, vient redoubler le récit proprement dit. Il assujettit explicitement la pérennisation du groupe social au sacrifice de certains de ses membres. L'ambition sociologique de Barrès consiste d'abord à illustrer par son récit et à expliquer par le discours, notamment dans le chapitre «La France dissociée et décérébrée», la crise traversée par la France contemporaine, minée par le déracinement. En racontant, sans se priver de la commenter, suivant la logique propre au roman à thèse, la manière dont cette crise conduit nécessairement à sacrifier des individus, transformés de fait en victimes expiatoires, il met au jour ce même processus que Durkheim va bientôt analyser dans le contexte de l'Affaire. La mise en évidence d'un mécanisme social participe néanmoins d'une autre logique que le discours du sociologue. Là où celui-ci remonte de façon inductive à la crise pour expliquer la persécution, le romancier présente le sacrifice comme la conséquence juste de la crise, au plan social sinon au plan moral, et comme le bon moyen de la dépasser. Il ne s'agit pas pour lui de démonter les rouages du mécanisme pour montrer le tort fait à des innocents, mais bien de justifier 
le mécanisme sacrificiel. Barrès présente certes Racadot et Mouchefrin comme les représentants d'un groupe minoritaire et comme des parias, mais il n'en souligne pas moins le bénéfice social de leur élimination. La question de l'innocence de Racadot ne compte pas en regard de ce qu'apporte à la société son élimination - comme l'innocence de Dreyfus, pour Barrès, en regard de la nécessité de ne pas affaiblir l'Armée ni la Nation - car comme le rappelle Roemerspacher, véritable porte-parole de l'auteur, «l'individu n'est rien, et la société tout» $(D, 615)$, l'individu lui-même n'étant qu'un des multiples organismes qui composent le corps social.

Dans ce roman qui peut apparaître comme le fruit du croisement entre le roman expérimental naturaliste et la sociologie, le mécanisme émissarial tend en effet à être présenté comme le résultat d'une loi naturelle, car l'ambition sociologique de Barrès ne l'empêche pas d'emprunter sa terminologie et certains schémas de pensées au discours médical ou biologique. Dans la perspective organiciste qui est celle de l'écrivain nationaliste, la société n'est qu'un organisme voué à éliminer ses cellules malades ou ses corps étrangers. Racadot et Mouchefrin n'ont pas encore commis leur crime, que leurs camarades plus fortunés du lycée de Nancy se détournent d'eux. De ce phénomène social, l'auteur rend compte par la formulation d'une loi générale, qui annonce la description du «mécanisme instinctif» de toute «collectivité» $(D, 686)$ : «Comme une basse-cour se rue sur un poulet malade pour l'achever ou l'expulser, chaque groupe tend à rejeter ses membres les plus faibles» $(D, 562)$. Comme le note par ailleurs Roemerspacher, «Ce Racadot, ce Mouchefrin, sont des poussières vénéneuses; il ne faut pas qu'ils se répandent pour tout empoisonner...» $(D, 725)$. Recouvrer l'intégrité, pour l'organisme social, suppose de retrancher, d'amputer: il s'agit donc de procéder à un épurement, suivant le terme présent dans tous les romans de Barrès, dès Le Culte du Moi. La référence à une supposée loi naturelle permet donc au romancier d'affirmer la nécessité d'un mécanisme social propre à guérir l'organisme, en sacrifiant certains individus: en ce sens, le nationalisme barrésien se donne aussi, comme la doctrine de Maurras, pour une «politique naturelle» ${ }^{15}$.

Loin de poursuivre une visée démystificatrice, comme le fait Durkheim lorsqu'il utilise le schéma émissarial pour expliquer l'antisémitisme, Barrès, pour sa part, ne fait rien d'autre que construire une mythologie, même si son discours adopte une tournure scientifique. Deux événements majeurs contribuent en effet à sacraliser le processus même d'émissarisation: la mort d'Astiné Aravian et la mort de Victor Hugo. L'assassinat d'Astiné n'est pas seulement l'acte qui rend possible légalement l'expulsion de Racadot et de Mouchefrin hors de l'organisme social, suivant un processus déjà en branle et expliqué dès le chapitre $V$ par la métaphore de la «bassecour» et du «poulet malade». Dans sa démarche qui vise à substituer progressivement à une perspective morale une perspective sociale, donnée pour positive, Barrès peut s'appuyer, consciemment ou non, sur les acquis de la pensée sociologique. Dans Les Règles de la méthode sociologique, Durkheim lui-même, trois ans plus tôt, a présenté le crime comme «un facteur de la santé publique, une partie intégrante de toute société saine» ${ }^{16}$. Considérant lui aussi le crime du strict point de vue de sa fonction

(15) «La politique naturelle» est le titre de la préface de Maurras à Mes Idées politiques; elle «consiste à reconnaître les faits de nature, à recueillir le legs du passé, à cerner les constances sociales» (G. LEROY, Les Écrivains et l'bistoire 1919-1956, Paris, Nathan, 1998, p. 19; sur la doctrine maurrassienne, voir plus généralement,pp. 18-21).

(16) Voir É. DuRkheim, Le crime, phénomène normal, dans Les Règles de la méthode sociologique (1894), Paris, Presses Universitaires de France, 1960, pp. 65-72. Durkheim, qui revient dans ce texte sur une «évidence paradoxale», à savoir que «le crime est normal», tire ces conclusions: «Classer le crime parmi les phénomènes de sociologie normale, ce n'est pas seulement dire qu'il est un phénomène inévitable quoique 
sociale, Barrès l'inscrit dans un contexte plus large qui conduit finalement à l'englober dans le schéma émissarial. La victime, l'Orientale Astiné, a en effet raconté sa vie à Sturel; elle lui a présenté ainsi une «coupe de poison» pleine de paroles susceptibles de «diffuser leurs dangereux éléments dans cet organisme en désordre» $(D, 553)$, tout en introduisant dans la «conscience» du jeune homme «comme un virus dans son sang, un principe par quoi devait être gâté son sens naturel de la vie» $(D, 553)$.

Les «circonstances tragiques» de la mort d'Astiné sont donc bien «de nécessité» $(D, 706)$, comme il est souligné dans le récit de cette mise à mort. La mise à mort d'Astiné est inscrite dans une chaîne de causalité rigoureuse: avant d'être eux-mêmes éliminés, Racadot et Mouchefrin, ces deux «poussières vénéneuses» $(D$, 725), éliminent l'Orientale qui a empoisonné moralement Sturel. Présenté comme le résultat d'un processus naturel, Barrès recourant au vocabulaire de «l'organisme» et des «énergies partielles» $(D, 705)$ pour en rendre compte, l'acte est hors de l'histoire; il s'inscrit dans un ordre mythique: «ce cadavre, ce sang et ces beautés découvertes, dans ce tragique abandon, c'est l'éternelle Hélène [...] qui une fois encore est venue du rivage homérique» $(D, 706)$. Plus tard dans le roman, les funérailles de Hugo, qui, au plan symbolique, achèvent le processus sacrificiel, en permettant à Sturel de baigner à nouveau dans le flot national, constituent un rite social répondant à la nécessité de créer «un Dieu d'accord avec un groupe important de l'humanité», afin de «maintenir l'unité, la fraternité française» $(D, 709)$. C'est pénétré des «mots de Hugo» dont la «force mystique agit sur notre organisme» $(D, 729)$ que Sturel reconsidère le meurtre d'Astiné par Racadot et Mouchefrin. Le chapitre consacré aux funérailles de Hugo se referme ainsi sur la célébration du culte des morts, par lequel l'individu peut s'intégrer à la collectivité en prenant conscience du «génie de notre race» $(D, 736)$.

Même si la représentation en est essentiellement négative, voire polémique, c'est un même mécanisme d'émissarisation, articulant le sacrifice d'un individu et la sauvegarde de la collectivité, qui s'inscrit au cœur de Leurs figures. La reconstruction de l'histoire observée par Sternhell ${ }^{17}$ joue ici à plein. S'il est question dans le roman du scandale de Panama, c'est bien l'Affaire qui donne un sens à cette représentation du parlementarisme. À plus forte raison est-ce le cas lorsque Barrès présente explicitement le baron Jacques de Reinach, tout prêt d'être lâché par les parlementaires corrompus, comme un bouc émissaire: «Nul doute qu'à cette extrémité [...] le baron de Reinach ne se comprît comme une victime expiatoire. [...] tout chassait ce malheureux dans les situations extrêmes. Ainsi Israël jadis poussait au désert le bouc chargé des malédictions qu'il fallait détourner de dessus le peuple» ( $L f, 1101)$. Pour sa part, Reinach endosse les malédictions ou les turpitudes du petit monde parlementaire, qui s'est enrichi grâce au Panama: en mettant fin à ses jours, l'individu peut espérer sauver le groupe. Un peu plus tard, c'est au tour des ministres de reproduire le mécanisme sacrificiel, en poursuivant le même but: «Ils jugèrent que la situation exigeait des sacrifices. Responsables du salut commun, ils décidèrent de livrer quelques camarades. [...] Ces hauts médecins décidèrent de procéder à l'amputation brutalement, avec une cruauté chirurgicale, car, par rapport au groupe doué du pouvoir de se reproduire, l'individu ne compte pas» (Lf, 1121).

Barrès reprend alors le vocabulaire médical, omniprésent dans Les Déracinés; en particulier, il utilise à différentes reprises l'image de «l'amputation» (Lf, 1127,

regrettable, dû à l'incorrigible méchanceté des hommes; c'est affirmer qu'il est un facteur de la santé publique, une partie intégrante de toute société saine» (p. 66).

(17) Voir supra, et note 8. 
1133). Au demeurant, dans Les Déracinés, Barrès avait déjà exposé le rôle joué par la Compagnie de Panama et mis en scène une rencontre entre Racadot, propriétaire du journal La Vraie République, et Reinach, susceptible de payer pour assurer le soutien du journal à la Compagnie ( $D, 667-669)$. La manière dont Barrès décrit l'élimination du député Baïhaut renvoie également au premier volet de la trilogie: son élimination est présentée comme un «étranglement» obtenu par le «supplice du garrot», ce qui évoque l'exécution de Racadot à la fin des Déracinés. De même, pour commenter la manière dont, «sur Baïhaut terrassé, tous ses amis se jetèrent», il reprend l'image frappante, utilisée dans Les Déracinés: «On eût dit d'un poulailler qui se rue sur un poulet malade, mais c'était plus réfléchi: ils voulaient, en le dévorant, faire disparaître celui qui le compromettait» $(L f, 1138)$. La précision est néanmoins importante, puisqu'elle marque toute la différence qui sépare l'élimination de Racadot de celle de Bailhaut ou de Reinach. Certes, le mécanisme émissarial aboutit à préserver le groupe, mais en l'occurrence il s'agit d'un groupe que Barrès considère comme corrompu, malsain et responsable au moins pour partie de la décadence nationale. Le sacrifice de la victime expiatoire n'est en l'occurrence qu'une parodie du sacrifice célébré dans Les Déracinés. Le mécanisme est identique, mais les fins en sont contraires: là où il s'agissait de restaurer l'intégrité de la nation, en éliminant des empoisonneurs, il s'agit maintenant de maintenir le parlementarisme, qui empoisonne cette même nation. L'ironie et l'amertume de Barrès ne font aucun doute. Ecrivant la chronique du scandale de Panama quelque dix ans après les faits, il en connaît l'issue: or si lui-même comptait parmi ceux qui espéraient du scandale qu'il porte un coup fatal au régime, on sait historiquement qu'il n'en fut rien, la crise ministérielle ayant effectivement rempli une fonction cathartique ${ }^{18}$.

Plus que le scandale du Panama, c'est pourtant l'Affaire qui donne la clef de ce portrait du baron de Reinach en bouc émissaire. La référence biblique à «Israël qui pousse au désert le bouc chargé des malédictions» n'est qu'une manière pour l'écrivain nationaliste de souligner le point crucial, à savoir la judéité de Reinach. C'est elle qui le conduit à devenir un bouc émissaire, sacrifié pour le bien du groupe: «puisque Joseph, sous sa redingote de la Conférence Molé, cache les obstinations d'un prophète d'Israël, j'admets que ce baron se sacrifia comme patriarche pour sa tribu» ( $L f$, 1102). Dans la perspective de Durkheim, c'est parce qu'il était juif que Dreyfus était devenu à son corps défendant une «victime expiatoire»:

Quand la société souffre, elle a besoin de trouver quelqu'un à qui elle puisse imputer son mal, sur qui elle se venge de ses déceptions; et ceux-là sont naturellement désignés pour ce rôle auxquels s'attache déjà quelque faveur de l'opinion. Ce sont les parias qui servent de victimes expiatoires. Ce qui me confirme dans cette interprétation, c'est la manière dont a été accueillie, en 1894, l'issue du procès Dreyfus. Ce fut un élan de joie sur les boulevards. [...] On savait donc enfin à qui s'en prendre du trouble économique et de la détresse morale où l'on vivait. C'est des juifs que venait le mal. Le fait était officiellement constaté19.

Pour Barrès, Reinach accepte volontairement de jouer ce rôle de victime expiatoire, en se suicidant pour préserver un groupe ou un corps, le corps parlementaire corrompu: en tant que juif, il se sacrifie en quelque sorte pour «sa tribu»; il agit ainsi

(18) Voir J.-M. MaYeur, Les Débuts de la Troisième république, Paris, Seuil, 1973, «Points», pp. 205-207; Mayeur observe précisément: «la crise ministérielle joua une fois de plus son rôle cathartique» (p. 207).

(19) É. DuRKHEIM, «Antisémitisme et crise sociale», repris dans Textes, vol. II, Paris, Éditions de Minuit, 1975 , p. 253. Le texte de Durkheim a été publié à l'époque dans H. DAGAN, Enquête sur l'antisémitisme, Paris, Stock, 1899. 
pour préserver un corps parlementaire essentiellement corrompu, voué à nourrir les parasites sans racines que sont le juif Reinach et le juif Cornelius Herz. Dans Les Déracinés, Reinach n'était-il pas déjà présenté comme un «fameux, influent et actif banquier juif, [...] un produit de la République parlementaire»? $(D, 626)$.

Dans l'ombre de Reinach, présenté dans Leurs figures comme la «victime expiatoire» de Panama, se devine la «victime expiatoire» Dreyfus, ainsi désigné par Durkheim dans le cadre de réflexions sur l'antisémitisme. Chacun connât l'observation de Barrès, en marge du procès de Rennes: «Je n'ai pas besoin qu'on me dise pourquoi Dreyfus a trahi. En psychologie, il me suffit de savoir qu'il est coupable de trahir et il me suffit de savoir qu'il a trahi. L'intervalle est rempli. Que Dreyfus est capable de trahir, je le conclus de sa race» ${ }^{20}$. Coupable, lui aussi, Reinach l'est également en raison de sa «race». Si l'inscription du schéma émissarial apparaît d'abord différent dans Les Déracinés et dans Leurs figures, ces deux représentations ne s'opposent pourtant pas, bien au contraire. La cohérence idéologique tient ici à la vision organiciste de la nation qui la sous-tend: après avoir célébré le sacrifice nécessaire des cellules malades dans Les Déracinés, Barrès affirme dans Leurs figures le sacrifice nécessaire des corps étrangers. La démonstration est d'autant plus efficace que dans le premier roman, il présentait déjà Reinach non seulement comme un Juif mais comme un «Allemand naturalisé», dont un des «frères, demeuré Allemand, dirige encore, à Francfort, la banque à la tête de laquelle mourut leur père en $1879 »(D, 627)$. Quand Durkheim explique l'antisémitisme, Barrès s'emploie pour sa part à le justifier, en recourant à la même figure émissariale que le sociologue, sauf qu'il substitue à l'image du juif «paria» celle du juif profiteur.

Dans la construction d'un discours nationaliste structuré autour des «grandes dichotomies» relevées par Susan Suleiman dans Le Roman de l'énergie nationale, «nationalisme vs. cosmopolitisme, traditionalisme vs. déracinement, patriotisme vs. égotisme, énergie collective vs. gaspillage nationaliste» ${ }^{21}$, le programme du bouc émissaire occupe donc une place privilégiée, liée sans doute à sa double nature. En tant que mécanisme cognitif reposant sur un modèle potentiellement narratif, il recouvre les deux dimensions ou les deux ambitions comportées par le genre du roman à thèse: raconter et expliquer, voire démontrer. Il s'accorde à l'ambition idéologique d'un romancier qui prétend écrire en sociologue tout en revendiquant une dimension scientifique, ce qui le conduit à être particulièrement réceptif au discours sociologique contemporain. S'il contribue à l'affirmation littéraire de la doctrine nationaliste - sinon à la «naissance littéraire du fascisme», selon la formule d'Uri Eisenzweig ${ }^{22}-$, ce n'est pourtant pas en raison des potentialités narratives qu'il comporte, même si celles-ci ne sont pas négligeables. Dans Les Déracinés, le schéma émissarial noue efficacement les différents fils narratifs autour de la question du déracinement (la liaison entre Sturel et Astiné Aravian, la faillite du journal monté par Racadot). Le programme du bouc émissaire présente également pour Barrès l'avantage de donne

(20) M. Barrès, À Rennes, dans Scènes et doctrines du nationalisme, Paris, Juven, 1902, p. 152.

(21) Voir S. Suleiman, op. cit., p. 148.

(22) Voir U. EIsenzweIG, Naissance littéraire du fascisme, Paris, Seuil, 2013, «La Librairie du XXI e siècle», en particulier la première des trois études rassemblées dans le volume, «Barrès: l'engagement», pp. 9-74; le mot fascisme est pris par Eisenzweig dans une acception large, le «marqueur de tout fascisme» mis en exergue correspondant rigoureusement à la définition du nationalisme: «une vision organique de la Nation à laquelle correspondent un refus de l'universel quant aux valeurs et un déterminisme racial quant à l'identité individuelle» (p. 7). L'idée même d'un «fascisme français» - qui serait, dans la perspective de Z. Sternhell, l'héritage du nationalisme de Barrès - fait débat chez les historiens: voir S. BERNSTEIN et M. WINOCK (éd.), Fascisme français, la controverse, Paris, CNRS Éditions, 2014. 
une déclinaison narrative et un prolongement politique aux thématiques majeures qui structurent ses romans, dès l'époque du Culte du Moi. Alors que le roman barrésien se déploie autour d'un idéal d'intégrité, essentiellement statique, donc antinarratif, recherché dans le moi d'abord, puis dans la nation, le schéma émissarial permet à Barrès de mettre en scène, en racontant ses divers épisodes (l'élimination d'Astiné, l'élimination de Racadot), la recomposition d'une intégrité menacée, dans un contexte sacrificiel qui tend à sacraliser la nation. Le récit participe ainsi directement à l'élaboration d'une mystique de la nation. La «fraternité», la «communion» et l'unité retrouvée de la foule française lors des funérailles de Victor Hugo constituent la face lumineuse du nationalisme barrésien. Le sacrifice des corps étrangers et des cellules malades, expulsés de l'organisme au terme d'un processus d'épuration donné pour la condition même de cette communion, désigne cependant le point le plus sombre de ce nationalisme. 